\title{
Application of a New Anatomic Hook-rod-pedicle Screw System in Young Patients With Lumbar Spondylolysis: a Pilot Study
}

\section{Duanming Li}

Chinese PLA General Hospital

\section{Wei Jiang}

Chinese PLA General Hospital

Baogan Peng ( $\sim$ pengbaogan@163.com )

Chinese PLA General Hospital

\section{Research Article}

Keywords: Lumbar spondylolysis, Hook-rod-pedicle screw system, Internal fixation, Bone healing

Posted Date: August 4th, 2021

DOI: https://doi.org/10.21203/rs.3.rs-764112/v1

License: (c) (i) This work is licensed under a Creative Commons Attribution 4.0 International License.

Read Full License 


\section{Abstract}

Background: Pedicle screw-laminar hook system has strong fixation and is conducive to bone graft fusion for lumbar spondylolysis. However, the current pedicle screw-laminar hook fixation system is not specifically designed for lumbar spondylolysis.

Methods: We designed a new anatomic hook-rod-pedicle screw system for young patients with lumbar spondylolysis. The isthmus and the corresponding pedicle screw entry point were exposed through intermuscular approach. Autogenous iliac bone graft was obtained to bridge the isthmus defect, and then anatomic hook-rod-pedicle screw system was used to fix the isthmus in 15 young patients.

Results: At 24 months follow-up, the Visual analogue scale (VAS) score of low back pain decreased from $6.73 \pm 0.88$ to $0.73 \pm 0.59$, and the Oswestry disability index (ODI) score decreased from $58.20 \pm 8.99$ to $7.87 \pm 4.97$. Computed tomography (CT) showed bilateral isthmic bone healing in 14 cases and unilateral isthmic bone healing in 1 case. Magnetic resonance imaging (MRI) showed that the lumbar disc signal of diseased segment and adjacent segments had no change compared with that before surgery. The pain VAS score of donor site was $0.20 \pm 0.41$ at the last follow-up. According to Macnab score, the excellent and good rate was $100 \%$.

Conclusions: The application of this new anatomical hook-rod-pedicle screw system to treat young patients with lumbar spondylolysis has the advantages of less trauma, simple operation and satisfactory clinical effects.

\section{Background}

Spondylolysis is a bony defect in the pars interarticularis of a vertebra, ${ }^{1,2}$ which can be complete or incomplete, bilateral or unilateral, and more commonly complete bilateral (Fig. 1 ). ${ }^{3}$ It is often asymptomatic but quite common in young people and adolescents with low back pain (LBP). ${ }^{4,5}$ These patients are usually treated conservatively with analgesics, lumbar orthoses, limitation of movement, and physical therapy, and surgery is performed only when the pain persists. ${ }^{6,7}$ For this young group of patients, spinal surgeons have paid more and more attention to how to minimize the impact on the range of motion of the spine and prevent the adjacent segments from producing excessive mechanical stress, 8,9 so as to turn their attention to repairing the pars interarticularis, bone grafting and restoring the stability of the posterior arch. ${ }^{10,11}$ Because the most common lesion of spondylolysis is acquired pseudarthrosis, bone grafting combined with internal fixation is a treatment that does not require arthrodesis. There are multiple reports on direct pars repair techniques in the literature. ${ }^{12-15}$ Two common methods are (1) direct repair using a lamina/pars compression screw through the isthmic defect; and (2) compression of the isthmic defect using a set of pedicle screw, rod, and laminar hook assembly within the same segment. 
More and more surgeons ${ }^{16-18}$ repair lumbar isthmus defect with bone grafting and pedicle screwlaminar hook system to treat young patients with lumbar spondylolysis, because of its firm fixation and good clinical effect. However, the pedicle screw-laminar hook system currently in use is not specifically designed for the treatment of lumbar spondylolysis. It has some disadvantages, such as incomplete matching between hook and lamina, difficulty in installation between rod and pedicle screw and large trauma. Complete exposure of the lamina is usually required, resulting in excessive paraspinal soft tissue dissection. To this end, we designed a new anatomical hook-rod-pedicle screw system and used it to treat 15 young patients with lumbar spondylolysis and to observe its clinical efficacy.

\section{Methods}

From April 2017 to July 2018,15 men with an average age of 22 (18-30 years) participated in the study. There were 11 cases of single segment, including L4 1 cases and L5 10 cases; 4 cases of double segments, L3 and L5 1 cases, and L4 and L5 3 cases. All cases were bilateral isthmus defects. Inclusion criteria: 1 . The patient presented with severe low back pain, limited lumbar function, but no radiating pain (sciatica) in the lower extremities. 2. Computed tomography (CT) of the lumbar spine showed spondylolysis, but no spina bifida or missing lamina; Dynamic lumbar radiographs showed no lumbar instability and spondylolisthesis. 3. At isthmic defect and adjacent levels, there was no disc degeneration on magnetic resonance imaging (MRI). 4. Conservative treatment, such as restriction of movement, oral anti-inflammatory analgesics and physiotherapy for 3-6 months, did not relieve symptoms. 5. Positive diagnosis test (low back pain disappeared after injection of small dose local anesthetics into the isthmus defect site). Exclusion criteria: metabolic diseases or chronic inflammatory diseases, such as arthrolithiasis, rheumatoid arthritis or ankylosing spondylitis. Physical examination revealed limited lumbar motion, tenderness above and/or adjacent spinous processes, and normal motor, sensory, and tendon reflexes in both lower extremities.

The study was approved by the ethical review committee from the General Hospital of Chinese Armed Police Forces and the study was in accordance with the Helsinki Declaration. All patients gave informed written consent.

The patient was placed prone on the operating table under general endotracheal anesthesia, carefully cushioning all pressure points and keeping the neck in a neutral position. A midline incision was made, cutting the skin to the deep fascia layer. The deep fascia was cut longitudinally $1.5 \mathrm{~cm}$ outside the midline. The longissimus-multifidusmuscle interval was bluntly dissected with a finger to avoid unnecessary tissue damage. Through intermuscular approach, the isthmic defect site and the insertion point of pedicle screw were exposed, and the fibrous tissue at the defect area was removed. A high-speed burr was used to debride sclerotic surfaces until bleeding bone surface was seen in the fractured pars. Gross motion was noted in the fissure area of the isthmus. Care was taken not to injure the facet joint capsule. Then a universal multiaxial pedicle screw was inserted into the corresponding vertebral body. Autogenous bone graft was obtained from the posterior superior iliac crest and implanted into the isthmus defect site, and the donor area of the posterior superior iliac crest was filled with allogeneic bone. 
After releasing the lower edge of the lamina with the ligamentum flavum stripper, the middle part of the hook rod was clamped with the rod holding forceps, and the hook could easily hook the lower edge of the lamina. The rod end of the anatomical hook was connected with the multiaxial pedicle screw. The construct was then loaded with compression force and tightened.

The contra-lateral anatomical hook-rod-pedicle screw was installed in the same way. After the installation of both sides, there was no loosening of hook-rod-pedicle screw system and no movement of bone graft. Then the wound was rinsed with saline, the drainage tube was placed, and the incision was closed layer by layer. The average intraoperative blood loss was $40 \mathrm{ml}(28-56 \mathrm{ml})$. On the second day after the operation, the drainage tube was pulled out, and patient put on lumbar brace and got out of bed. X-ray, CT and MRI were reexamined at $3,6,12$ and 24 months after operation.

\section{Results}

During the follow-up period of 24 months, no patient developed sciatica, motor or sensory disturbance. At the final follow-up, the VAS score of low back pain decreased from $6.73 \pm 0.88$ preoperatively to $0.73 \pm$ 0.59 postoperatively, and the ODI score decreased from $58.20 \pm 8.99$ preoperatively to $7.87 \pm 4.97$ postoperatively (Table 1). CT showed bilateral isthmus bone fusion in 14 cases and unilateral isthmus bone fusion in 1 case. The signs of intervertebral discs in diseased and adjacent segments had no change on MRI. The VAS score of donor site pain was $0.20 \pm 0.41$. Allogeneic bone filling in the bone donor site showed osteogenesis (Fig. 2). According to Macnab standard, ${ }^{19}$ the excellent and good rate was $100 \%$ (Table 2). Typical case was shown in Fig. 3.

Table 1

VAS and ODI scores in each time point

\begin{tabular}{|llllll|}
\hline Measure & Baseline & 3 Months & 6 Months & 12Months & 24Months \\
\hline Back Pain VAS & $6.73 \pm 0.88$ & $2.20 \pm 0.86$ & $1.47 \pm 0.92$ & $1.13 \pm 0.64$ & $0.73 \pm 0.59$ \\
\hline ODI & $58.20 \pm 8.99$ & $21.73 \pm 6.24$ & $16.40 \pm 4.55$ & $12.13 \pm 3.72$ & $7.87 \pm 4.97$ \\
\hline Donor area VAS & - & $1.93 \pm 0.96$ & $0.67 \pm 0.82$ & $0.40 \pm 0.51$ & $0.20 \pm 0.41$ \\
\hline
\end{tabular}

Table 2

Macnab rating in different time points

\begin{tabular}{|lllll|}
\hline Postoperative Time & Excellent & Good & Fair & Poor \\
\hline 3 months & $5(33 \%)$ & $7(47 \%)$ & $3(20 \%)$ & 0 \\
\hline 6 months & $6(40 \%)$ & $8(53 \%)$ & $1(7 \%)$ & 0 \\
\hline 12 months & $8(53 \%)$ & $7(47 \%)$ & 0 & 0 \\
\hline 24 months & $10(67 \%)$ & $5(33 \%)$ & 0 & 0 \\
\hline
\end{tabular}




\section{Discussion}

Lumbar spondylolysis is one of the common causes of low back pain in adolescents. ${ }^{4}$ The incidence rate is $3-10 \%$ in adolescents and $6 \%$ in adults. ${ }^{20,21}$ More than $80 \%$ of lumbar spondylolysis appear in L4 and L5. ${ }^{4}$ Patients with lumbar spondylolysis mostly like sports or engage in sports, dancing and other industries. The specific cause of spondylolysis may be stress fractures caused by long-term fatigue on the basis of isthmic dysplasia. For the treatment of symptomatic lumbar spondylolysis in adolescents, active measures should be taken to avoid further problems such as intervertebral disc degeneration, herniation, lumbar instability or spondylolisthesis. If early diagnosis of lumbar spondylolysis is made in adolescents, measures such as wearing a lumbosacral brace and restricting movement will most likely result in isthmic healing, ${ }^{22}$ but those who do not heal should be actively treated by surgery. Main indication for surgical repair of lumbar spondylolysis is that low back pain is not relieved after at least 6 months of non-surgical treatment, including activity modification, bracing, and physical therapy. Aggravation of pain, deterioration of neurological symptoms and progressive listhesis also are indications for surgical consideration. In the present study, our patient group, due to severe low back pain, failure of conservative treatment for more than 3 months, isthmus dissection, osteosclerosis and nonunion, needed surgical treatment.

There are many surgical methods for lumbar spondylolysis. In 1968, Kimura described an isolated bone graft that directly repaired the isthmus defect without internal fixation, retained segmental activity, but required postoperative cast and long bed rest. ${ }^{23}$ Later, Scott ${ }^{24}$ proposed the use of wire under the lamina and transverse processes, which has been improved by several authors over the years. ${ }^{25,26}$ In 1970, Buck $^{27}$ first used screw internal fixation and bone grafting to repair defects directly, and subsequently other approaches with special constructs and temporary fixations were reported. ${ }^{28,29}$ There were also posterolateral bone graft fusion, cross-segmental pedicle screw fixation and other methods. Patients with spondylolisthesis or disc herniation can be treated with pedicle screw fixation and interbody fusion. ${ }^{30}$

The treatment of young patients with lumbar spondylolysis with isthmus debridement, bone grafting, and pedicle screw laminar hook fixation has achieved satisfactory results, $17,18,31$ which proves that pedicle screw-laminar hook system has strong fixation and is conducive to bone graft fusion. It is an intrasegmental fixation and does not affect the lumbar interbody movement and the kinematics of the adjacent segment. Studies ${ }^{32-34}$ have reached a consensus that lumbar intramuscular approach can reduce the dissection of paravertebral muscles, reduce the denervation of paravertebral muscles, preserve the structure of muscle ligament complex, reduce postoperative pain and recover quickly. However, the current pedicle screw-laminar hook fixation system is not specifically designed for lumbar spondylolysis. Before the hook is installed, the muscles around the spinous process and lamina need to be separated, resulting in severe tissue damage. At the same time, the installation of the system is difficult because the lamina, hook and pedicle screw are not on the same plane. 
To solve these problems, we designed a new anatomical hook-rod instrument (Fig. 4), which combined with pedicle screw to form anatomical hook-rod-pedicle screw system. The system can be installed by intermuscular approach, which has the advantages of less trauma and convenient operation. At the same time, the system is firmly fixed, which is favorable for bone graft fusion. According to the anatomy of lumbar spine, the spinous process is at a certain angle with the lamina, the lamina is inclined backward and upward, and the lower edge of lamina and the tail of pedicle screw are at a certain angle with the sagittal plane. According to the above anatomical features, the hook and the rod are inclined in these three directions. The hook is completely matched with the lamina, which is conducive to the installation of the hook at the lower edge of the lamina, and the rod is easy to connect with the universal pedicle screw. Of course, the angles of L4 and L5 are different. We have designed a series of hook-rods with different angles, which are convenient for operation. In this study, 15 cases of young patients with lumbar spondylolysis were treated with isthmus repair, bone grafting, anatomical hook-rod-pedicle screw fixation, and achieved satisfactory results. At the same time, the injury was small, and the operation was simple and convenient.

Autologous iliac bone graft is the "gold standard" in bone grafting, ${ }^{35}$ and pain in the iliac bone donor area is a common complication after iliac bone removal. ${ }^{36}$ There are many reasons for postoperative pain in the donor area, such as bone defect, adhesion, osteoporosis and cutaneous nerve injury in the donor area. A bone block with cortex and cancellous bone is taken from the posterior superior iliac spine and can be trimmed to a suitable size to meet the needs of bone grafting in the isthmus. To solve the problem of donor site pain, we used the allogeneic bone with tissue-engineered human bone morphogenetic proteins (BMPs) to fill the defect area of posterior superior iliac spine. Allogeneic bone contains BMPs, which can induce new bone formation and promote bone growth. During the follow-up, bone growth was found in the defect of posterior superior iliac spine, as shown in (Fig. 2), and the pain in the bone donor area disappeared.

The application of isthmus debridement, bone grafting and anatomical hook-rod-pedicle screw system fixation in young patients with lumbar spondylolysis has the advantages of less trauma, simple operation and satisfactory curative effect. However, it is not suitable for the cases of lumbar spondylolysis with spondylolisthesis. In addition, it is not also suitable for the cases with missing lamina, bone dysplasia and lumbar disc degenerative diseases. This new hook-rod-pedicle screw system is undergoing biomechanical testing and has been patented in China (Patent No.: ZL201721043286.7). This is a small sample observation study, and further large sample and prospective studies are needed to prove the superiority and reliability of the system.

\section{Conclusions}

Compared with the use of traditional instrument, the application of this new anatomical hook-rod-pedicle screw system to treat young patients with lumbar spondylolysis has the advantages of less trauma, simple operation and satisfactory clinical effects. 


\section{Abbreviations}

LBP: Low back pain

CT: Computed tomography

MRI: Magnetic resonance imaging

VAS: Visual analogue scale

ODI: Oswestry disability index

BMP: Bone morphogenetic protein

\section{Declarations}

\section{Ethics approval and consent to participate}

All procedures performed in studies involving human participants were in accordance with the ethical standards of the institutional and/or national research committee and with the 1964 Helsinki declaration and its later amendments or comparable ethical standards. This study was approved by the ethics committee of The Third Medical Centre of Chinese PLA General Hospital. We obtained written informed consent from all participants before publishing this information.

\section{Consent for publication}

Written informed consent for publication was obtained from all participants.

\section{Availability of data and materials}

The datasets used and/or analyzed during the current study are available from the corresponding author on reasonable request.

\section{Competing interests}

All authors declare that they have no competing interests.

\section{Funding}

No funds were received in support of this study.

\section{Contributions}

Duanming Li and Wei Jiang designed the study. Baogan Peng wrote the manuscript text. Duanming Li and Baogan Peng performed the surgical operations. All authors reviewed the manuscript. The authors read and approved the final manuscript. 
Acknowledgements

Not applicable

\section{Author information}

Department of Orthopaedics (Duanming Li, Baogan Peng $\varangle ;$ Department of Anesthesiology (Wei Jiang); The Third Medical Centre of Chinese PLA General Hospital, Beijing, China

\section{Corresponding authors}

Correspondence to Wei Jiang or Baogan Peng

\section{References}

1. Farfan HF, Osteria V, Lamy C. The mechanical etiology of spondylolysis and spondylolisthesis. Clin Orthop Relat Res. 1976;117:40-55.

2. Wiltse LL, Newman PH, Macnab I. Classification of spondylolysis and spondylolisthesis. Clin Orthop Relat Res. 1976;117:23-29.

3. Chen MR, Moore TA, Cooperman DR, Lee MJ. Anatomic variability of 120 L5 spondylolytic dfects. Global Spine J. 2013;3:243-248.

4. Hensinger RN. Spondylolysis and spondylolisthesis in children and adolescents. J Bone Joint Surg Am. 1989;71:1098-1107.

5. Nachemson A. Repair of the spondylolisthesic defect and intertransverse fusion for young patients. Clin Orthop Relat Res.1976;117:101-105.

6. Blanda J, Bethem D, Moats W, Lew M. Defects of pars interarticularis in athletes: a protocol for nonoperative treatment. J Spinal Disord. 1993;6:406-411.

7. Steiner ME, Micheli LJ. Treatment of symptomatic spondylolysis and spondylolisthesis with modified Boston brace. Spine (Phila Pa 1976). 1985;10:937-943.

8. Lee CK. Accelerated degeneration of the segment adjacent to a lumbar fusion. Spine (Phila Pa 1976). 1988;13:375-377.

9. Yang SW, Langrana NA, Lee CK. Biomechanics of lombosacral spinal fusion in combined compression-torsion loads. Spine (Phila Pa 1976). 1980;11:937-941.

10. Cyron BM, Hutton WC. The fatigue strength of the lumbar neural arch in spondylolysis. J Bone Joint Surg Br. 1978;60-B:234-238.

11. Green TP, Allvey JC, Adams MA. Spondylolysis. Bending of the inferior articular processes of lumbar vertebrae during simulated spinal movements. Spine (Phila Pa 1976). 1994;19:2683-2691.

12. Hefti F, Seeling W, Morscher E. Repair of lumbar spondylolysis with a hook-screw. Int Orthop. 1992;16:81-85. 
13. Pavlovcic V. Surgical treatment of spondylolysis and spondylolisthesis with a hook screw. Int Orthop. 1994;18:6-9.

14. Ohmori K, Suzuki K, Ishida Y. Translamino-pedicle screw fixation with bone grafting for symptomatic isthmic lumbar spondylolysis. Neurosurgery. 1992;30:379-384.

15. Gillet $P$, Petit M. Direct repair of spondylolysis without spondylolisthesis, using a rod-screw construct and bone grafting of the pars defect. Spine (Phila Pa 1976). 1999;24(12):1252-1256.

16. Tokuhashi Y, Matsuzaki H. Repair of defects in spondylolysis by segmental pedicle screw hook fixation. Spine (Phila Pa 1976). 1996;21:2041-2045.

17. Roca J, Iborra M, Cavanilles-Walker JM, Albertí G. Direct repair of spondylolysis using a new pedicle screw hook fixation: clinical and CT-assessed study: an analysis of 19 patients. J Spinal Disord Tech. 2005;18 Suppl:S82-89.

18. Debusscher F, Troussel S. Direct repair of defects in lumbarspondylolysis with a new pedicle screw hook fixation: clinical functional and Ct-assessed study. Eur Spine J.2007;16:1650-1658.

19. Macnab I. Negative disc exploration. An analysis of the causes of nerve-root involvement in sixtyeight patients. J Bone Joint Surg Am. 1971;53:891-903.

20. Rajasekaran S, Kamath V, Avadhani A. Bucks fusion. Eur Spine J. 2010;19:343-344.

21. Smith JA, Hu SS. Management of spondylolysis and spondylolisthesis in the pediatric and adolescent population. Orthop Clin North Am. 1999;30:487-499. ix.

22. Steiner ME, Micheli LJ. Treatment of symptomatic spondylolysis and spondylolisthesis with the modified Boston brace. Spine (Phila Pa 1976). 1985;10:937-943.

23. Kimura M. My method of filling the lesion with spongy bone in spondylolysis and spondylolisthesis. Orthop Surg (Jpn). 1968;19:285-295.

24. Askar Z, Wardlaw D, Koti M. Scott wiring for direct repair of lumbar spondylolysis. Spine (Phila Pa 1976). 2003;28:354-357.

25. Hambly MF, Wiltse LL.A modification of the Scott wiring technique. Spine (Phila Pa 1976). 1994;19:354-356.

26. Johnson GV, Thompson AG. The Scott wiring technique for direct repair of lumbar spondylolysis.J Bone Joint Surg Br. 1992;74:426-430.

27. Buck JE. Direct repair of the defect in spondylolisthesis: preliminary report.J Bone Joint Surg. 1970;52:432-437.

28. Morscher E, Gerber B, Fase J. Surgical treatment of spondylolisthesis by bone grafting and direct stabilization of spondylolysis by means of a hook screw. Arch Orthop Trauma Surg. 1984;103:175178.

29. Louis R. Pars interarticularis reconstruction of spondylolysis using plates and screws with grafting without arthrodesis. Apropos of 78 cases. Rev Chir Orthop Reparatrice Appar Mot. 1988;74:549-557.

30. Zhang S, Ye C, Lai Q, Yu X, Liu X, Nie T, Zhan H, Dai M, Zhang B. Double-level lumbar spondylolysis and spondylolisthesis: A retrospective study. J Orthop Surg Res. 2018; 13(1):55. 
31. Tokuhashi Y, Matsuzaki H. Repair of defects in spondylolysis by segmental pedicle screw hook fixation. Spine (Phila Pa 1976). 1996;21:2041-2045.

32. Vialle R, Wicart P, Drain O, Dubousset J, Court C. The Wiltse paraspinal approach to the lumbar spine revisited: an anatomic study. Clin Orthop Relat Res. 2006;445:175-180.

33. Suk KS, Lee HM, Kim NH, Ha JW. Unilateral versus bilateral pedicle screw fixation in lumbar spinal fusion. Spine (Phila Pa 1976). 2000;25:1843-1847.

34. Whiteclound TS, Roesch WW, Ricciardi JE. Transforaminal-interbody fusion verus anterior-posterior interbody fusion of the lumbar spine: a financial analysis. J Spinal Disord Tech. 2001; 14:100-103.

35. Dhawan A,Kuklo TR,Polly DW. Analysis of iliac crest bone grafting process measures. Am J Orthop. 2006;35:322-326.

36. Delawi D, Dhert WJ, Castelein RM, Verbout AJ, Oner FC. The incidence of donor site pain after bone graft harvesting from the posterior iliac crest may be overestimated: a study on spine fracture patients. Spine (Phila Pa 1976). 2007;32:1865-1868.

\section{Figures}




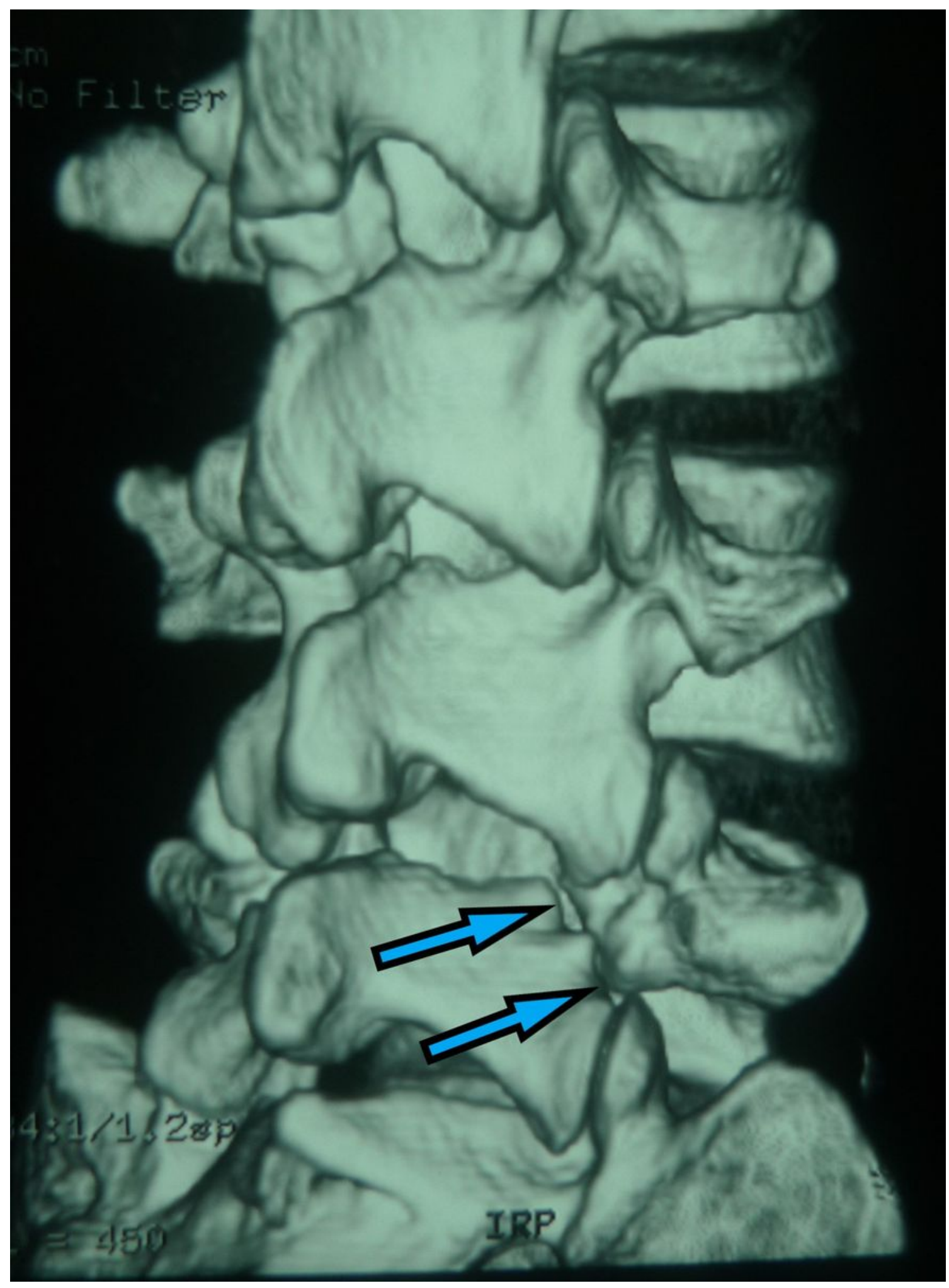

\section{Figure 1}

Three-dimensional CT scan showed L5 isthmus fracture (blue arrows). 


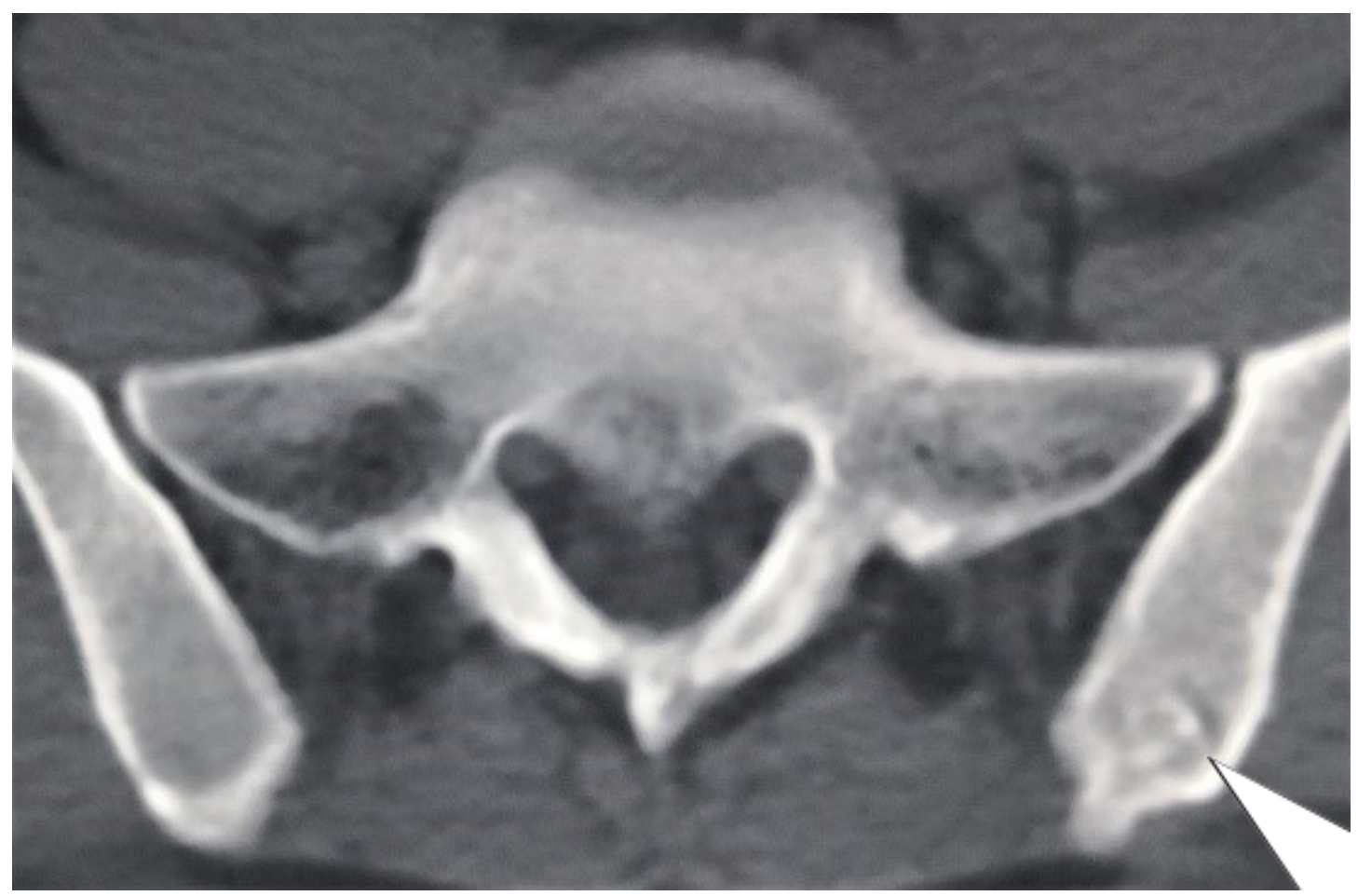

Figure 2

The donor site of iliac crest was filled with allogeneic bone, which resulted in osteogenesis (white arrow). 

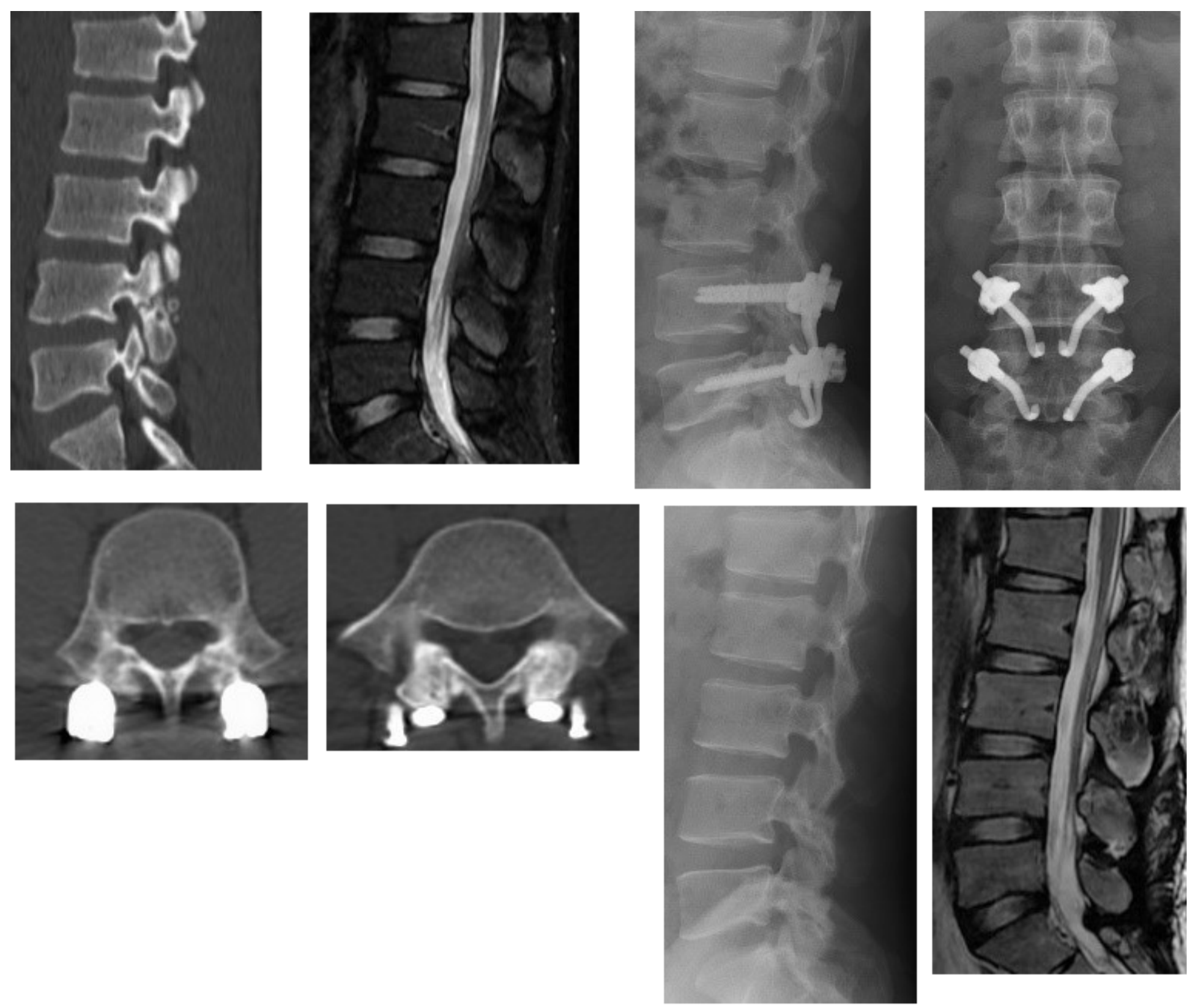

\section{Figure 3}

Typical case. A 21-year-old male patient had recurrent low back pain for more than 2 years. a. Twodimensional CT scan showed lumbar spondylolysis at bilateral L4 and L5 levels. b. Lumbar MRI showed normal signals of all lumbar intervertebral discs. c. Lateral radiograph after lumbar surgery. $d$. Anteroposterior radiograph of lumbar spine after operation. e. CT scan of lumbar spine at 6 months after lumbar operation showed the healing of bone graft in the L4 isthmus. f. CT scan of lumbar spine at 6 months after lumbar operation showed the healing of bone graft in the L5 isthmus. g. 12 months after the operation, the lateral radiograph showed that the internal fixation had been removed. h. 12 months after the operation, MRI showed that the signals of all lumbar intervertebral discs were normal. 

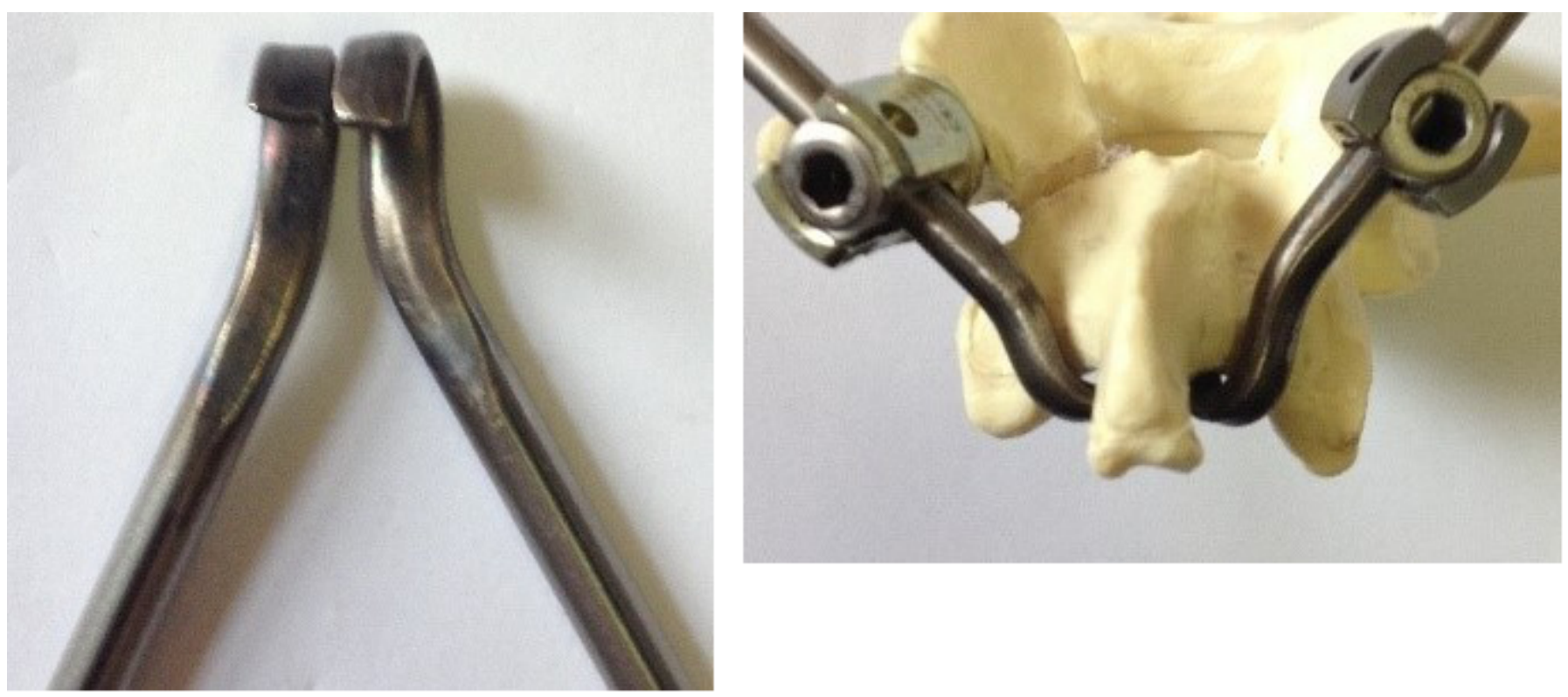

Figure 4

a. Newly designed anatomical hook with rod. b. The hook was completely matched with the lamina, and the rod was connected with the pedicle screw. 